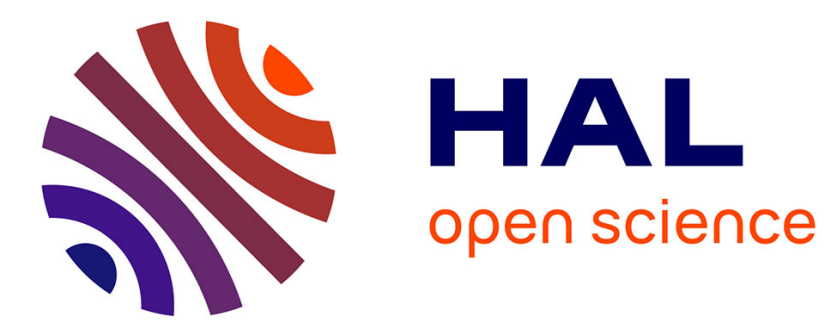

\title{
Femtosecond Laser Excitation of Spin Resonances in Amorphous Ferrimagnetic $\mathrm{Gd}(1-\mathrm{x}) \mathrm{Co}(\mathrm{x})$ Alloys
}

A. Mekonnen, M. Cormier, Av Kimel, A. Kirilyuk, Ales Hrabec, Laurent

Ranno, T. Rasing

\section{- To cite this version:}

A. Mekonnen, M. Cormier, Av Kimel, A. Kirilyuk, Ales Hrabec, et al.. Femtosecond Laser Excitation of Spin Resonances in Amorphous Ferrimagnetic Gd(1-x)Co(x) Alloys. Physical Review Letters, 2011, 107 (11), pp.117202. 10.1103/PhysRevLett.107.117202 . hal-00635142

HAL Id: hal-00635142

https://hal.science/hal-00635142

Submitted on 24 Oct 2011

HAL is a multi-disciplinary open access archive for the deposit and dissemination of scientific research documents, whether they are published or not. The documents may come from teaching and research institutions in France or abroad, or from public or private research centers.
L'archive ouverte pluridisciplinaire HAL, est destinée au dépôt et à la diffusion de documents scientifiques de niveau recherche, publiés ou non, émanant des établissements d'enseignement et de recherche français ou étrangers, des laboratoires publics ou privés. 


\title{
Femtosecond Laser Excitation of Spin Resonances in Amorphous Ferrimagnetic $\mathbf{G d}_{1-x} \mathbf{C o}_{x}$ Alloys
}

\author{
A. Mekonnen, ${ }^{1}$ M. Cormier, ${ }^{1}$ A. V. Kimel, ${ }^{1}$ A. Kirilyuk, ${ }^{1}$ A. Hrabec, ${ }^{2}$ L. Ranno, ${ }^{2}$ and Th. Rasing ${ }^{1}$ \\ ${ }^{1}$ Radboud University Nijmegen, Institute for Molecules and Materials, Heyendaalseweg 135, 6525 AJ Nijmegen, The Netherlands \\ ${ }^{2}$ Institut Néel, CNRS/UJF, 25 Rue des Martyrs, 38042 Grenoble Cedex 9, France
}

(Received 29 June 2011; published 8 September 2011)

\begin{abstract}
Using 100 fs laser pulses, a high-frequency exchange and a low-frequency ferromagnetic resonance mode have been excited and detected in an amorphous $\mathrm{Gd}_{1-x} \mathrm{Co}_{x}(78 \leq x \leq 85)$ ferrimagnetic thin film, on both sides of its angular momentum compensation composition. The excitation efficiency of these modes strongly depends on the amount of laser-induced heating. Ways of selectively and efficiently exciting either one or both of these two coexisting magnetic modes by adjusting laser pulse intensity are demonstrated.
\end{abstract}

DOI: 10.1103/PhysRevLett.107.117202

PACS numbers: 75.50.Gg, 75.50.Kj, 75.78.Jp, 76.50.+g

Exciting and controlling spins in a magnetically ordered system has become a rapidly evolving area of science in the past decade [1-10]. From the viewpoint of applications, magnetization reversal lies at the heart of all magnetic recording and information processing technologies. Fundamentally, magnetization reversal can be seen as the transfer of a spin system over a potential barrier separating two metastable states, which correspond to magnetizations "up" and "down", respectively. It has been demonstrated that one of the fastest ways to overcome this barrier and reverse the magnetization in a ferromagnetic medium is to excite the homogeneous mode of spin precession $[2,3,5]$ (ferromagnetic resonance), resulting in a reversal via a precessional motion of spins (typically on the time scale of 0.1 to $1 \mathrm{~ns})$. Very recently, a totally new route for magnetization reversal was discovered $[9,10]$ in a twosublattice ferrimagnetic alloy. In these experiments, excitation of the ferrimagnet by a subpicosecond laser pulse was shown to trigger a precession-free reversal within tens of picoseconds via a strongly nonequilibrium magnetic state with no net magnetization. The origin of this state and the way an ultrafast optical excitation can lead to such fast reversal are beyond the state-of-the-art theory of magnetism and still remain poorly understood. How does an ultrafast laser pulse pump energy into the ferrimagnetic system, and how is this energy used to achieve ultrafast reversal? What are the roles of the various spin resonance modes in these processes? These intriguing questions have remained largely unanswered so far.

In this Letter, we present our results on laser-induced spin dynamics in $\mathrm{Gd}_{1-x} \mathrm{Co}_{x}$ alloys by using an all-optical pump-probe technique. We show that a laser pulse is able to excite both ferromagnetic and exchange spin resonance modes. We reveal an interplay between these two channels of excitation, and find the conditions under which either one or both of these two magnetic modes can be efficiently excited in a controllable way.
For this study, we used an amorphous $\mathrm{Gd}_{1-x} \mathrm{Co}_{x}$ $(78 \leq x \leq 85)$ ferrimagnetic alloy thin film, grown by magnetron sputtering on a quartz substrate and sandwiched inbetween Ti layers: $\mathrm{Ti}(3 \mathrm{~nm}) / \mathrm{Gd}_{1-x} \mathrm{Co}_{x}(20 \mathrm{~nm}) / \mathrm{Ti}(3 \mathrm{~nm})$. This film has a concentration gradient, 1 at. \% Co/mm [11], along one direction of the sample and an out-of-plane magnetic anisotropy. In this alloy, the two different magnetic sublattices are coupled antiferromagnetically. Since the Co and Gd sublattice magnetizations depend differently on temperature, they can compensate each other at a certain temperature, $T_{M}$, where $M_{\mathrm{RE}}=M_{\mathrm{TM}}$. Such a system can also compensate its angular momentum, $M_{\mathrm{RE}} / \gamma_{\mathrm{RE}}=$ $M_{\mathrm{TM}} / \gamma_{\mathrm{TM}}$, where $\gamma$ is the gyromagnetic ratio, at another temperature known as the angular momentum compensation temperature $T_{A}$. Since the compensation temperatures [12], the magnetic anisotropies [13], and the effective damping parameters [14] depend on the composition of the sample, $\mathrm{Gd}_{1-x} \mathrm{Co}_{x}$ gives many degrees of freedom to investigate the influence of the multisublattice structure on its spin dynamics.

The equation of motion of a two-sublattice ferrimagnetic system can be written in the form of two coupled LandauLifshitz-Gilbert (LLG) equations [15,16]:

$$
\frac{d \mathbf{M}_{i}}{d t}=-\gamma_{i}\left(1+\alpha_{i}^{2}\right)\left(\mathbf{M}_{i} \times \mathbf{H}_{\mathrm{eff}}^{i}\right)+\frac{\alpha_{i}}{\left|M_{i}\right|}\left(\mathbf{M}_{i} \times \frac{d \mathbf{M}_{i}}{d t}\right),
$$

where $\alpha_{i}$ is the phenomenological Gilbert damping parameter, $\gamma_{i}>0$ is the gyromagnetic ratio, and $M_{i}$ is the saturation magnetization of the $i$ th sublattice $(i=\mathrm{Co}, \mathrm{Gd})$. The effective magnetic fields experienced by the Co and Gd sublattices can be written as

$$
\mathbf{H}_{\mathrm{eff}}^{\mathrm{Co}}=\mathbf{H}_{\mathrm{ext}}+\mathbf{H}_{\mathrm{Co}}^{\mathrm{a}}+\lambda_{\mathrm{Gd}-\mathrm{Co}} \mathbf{M}_{\mathrm{Gd}}
$$

and

$$
\mathbf{H}_{\mathrm{eff}}^{\mathrm{Gd}}=\mathbf{H}_{\mathrm{ext}}+\mathbf{H}_{\mathrm{Gd}}^{\mathrm{a}}+\lambda_{\mathrm{Gd}-\mathrm{Co}} \mathbf{M}_{\mathrm{Co}},
$$


where $\mathbf{H}_{\mathrm{ext}}$ is the externally applied magnetic field, $\lambda_{\mathrm{Gd}-\mathrm{Co}}<0$ is the intersublattice exchange coupling coefficient, $\mathbf{H}_{\mathrm{Co}}^{\mathrm{a}}$ and $\mathbf{H}_{\mathrm{Gd}}^{\mathrm{a}}$ are the magnetic anisotropy fields of the Co and Gd sublattices, and $\lambda_{\mathrm{Gd}-\mathrm{Co}_{0}} \mathbf{M}_{\mathrm{Co}}$ and $\lambda_{\mathrm{Gd}-\mathrm{Co}} \mathbf{M}_{\mathrm{Gd}}$ are the exchange fields created by the Co and Gd sublattices, respectively. The solution of Eq. (1) gives two homogenous modes of spin resonance. The first one is the ferromagnetic resonance (FMR) mode, the frequency of which is proportional to the effective magnetic field [17]:

$$
f_{\mathrm{FMR}}=\gamma_{\mathrm{eff}} \mathrm{H}_{\mathrm{eff}},
$$

where $\gamma_{\text {eff }}$ is an effective gyromagnetic ratio

$$
\gamma_{\mathrm{eff}}=\frac{x M_{\mathrm{Co}}-(1-x) M_{\mathrm{Gd}}}{x M_{\mathrm{Co}} / \gamma_{\mathrm{Co}}-(1-x) M_{\mathrm{Gd}} / \gamma_{\mathrm{Gd}}}
$$

( $x$ is the Co concentration). The second spin resonance mode is called exchange mode and its frequency is given by $[17,18]$ :

$$
f_{\text {exch }}=\lambda_{\mathrm{Gd}-\mathrm{Co}} \gamma_{\mathrm{Co}} \gamma_{\mathrm{Gd}}\left[x M_{\mathrm{Co}} / \gamma_{\mathrm{Co}}-(1-x) M_{\mathrm{Gd}} / \gamma_{\mathrm{Gd}}\right] \text {. }
$$

The frequencies of both modes thus depend on the relative concentrations of $\mathrm{Co}$ and $\mathrm{Gd}$ ions in the alloy. Because of the large value of the exchange fields, the frequency of the exchange mode, $f_{\text {exch }}$, normally falls in the infrared (THz) region. However, $f_{\text {exch }}$ can be brought down to the microwave $(\mathrm{GHz})$ region [19] if one works at (or in the vicinity of) angular momentum compensation (AMC), where $x M_{\mathrm{Co}} / \gamma_{\mathrm{Co}}=(1-x) M_{\mathrm{Gd}} / \gamma_{\mathrm{Gd}}$ [Eq. (6)]. Besides, from Eqs. (4) and (5), we see that at AMC the FMR frequency $f_{\mathrm{FMR}}$ diverges.

Laser-induced spin dynamics was investigated by an alloptical pump-probe experiment using an amplified pulsed Ti:Sapphire laser at $805 \mathrm{~nm}$ wavelength, with a pulse width of about $100 \mathrm{fs}$. The pulsed beam was split into two linearly polarized parts with unequal intensities. The stronger pump pulse was focused onto a spot with a diameter around $400 \mu \mathrm{m}$, and an incidence angle about $15^{\circ}$ from the sample normal. The weaker probe pulse, delayed by a certain time $t_{\text {delay }}$, was focused onto a smaller spot with a diameter around $50 \mu \mathrm{m}$ at normal incidence. The delay between pump and probe pulses was controlled by a delay line. The transmitted probe pulse was detected by a balanced-diode detector after polarization analysis by a Wollaston prism [see inset in Fig. 1(a)]. In these experiments, the magnetooptical signal mainly comes from the Co sublattice [20]. Unless otherwise specified, all the experiments were performed at room temperature (i.e., far below the Curie temperature $T_{\mathrm{C}}[21,22]$ which is the same for both sublattices [23]), under an external magnetic field of $0.27 \mathrm{~T}$ applied at an angle $\approx 70^{\circ}$ from the sample normal, and with a pump-laser fluence of $10 \mathrm{~mJ} / \mathrm{cm}^{2}$.

With the external field at an oblique angle, laser-induced heating causes a change in the temperature-dependent
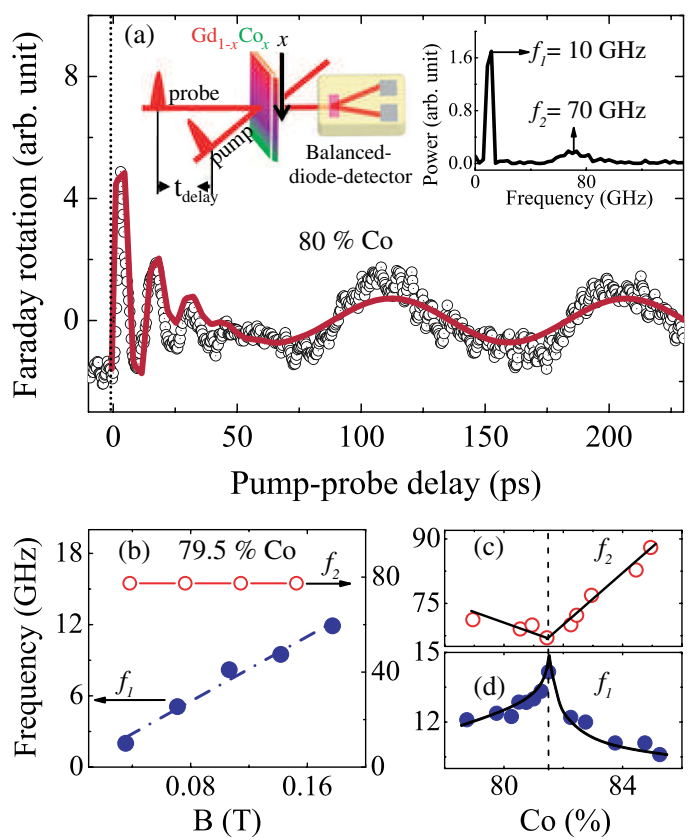

(e)

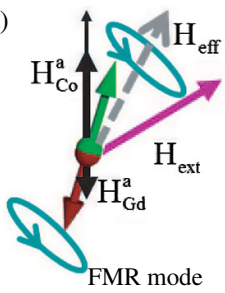

(f)

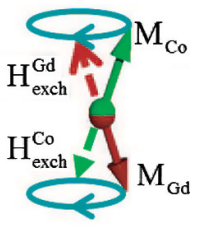

Exchange mode

FIG. 1 (color online). (a) Optically induced magnetic oscillations for $80 \%$ Co. The vertical dotted line indicates zero delay between pump and probe pulses and the solid curve is a fit using Eq. (7). The experiment schematic and the fast Fourier transform (FFT) spectrum of the oscillations are shown as insets. (b) Oscillation frequencies for $79.5 \% \mathrm{Co}$, as a function of the external magnetic field applied at $\approx 55^{\circ}$ from the sample normal. The dash-dotted line is a fit using Eq. (4). (c)-(d) Oscillation frequencies as a function of cobalt concentration (symbols). Solid lines are guides to the eye. (e)-(f) Schematics that show the precession of the magnetizations around (e) the effective field $\mathbf{H}_{\text {eff }}$ and (f) the exchange fields $\mathbf{H}_{\text {exch }}^{\mathrm{Co}, \mathrm{Gd}}$, which give rise to the FMR and exchange modes, respectively.

magnetization values, and thus a sudden change in the effective magnetic fields, triggering a precessional motion of the spins.

The observed spin dynamics for $80 \%$ Co is plotted in Fig. 1(a). We clearly see two different dynamical features with different oscillation periods. The first one with a short period dominates in the first $50 \mathrm{ps}$, followed by the second one with a longer period. This is confirmed by the fast Fourier transform (FFT) spectrum of these data, shown as inset in Fig. 1(a), where two well-resolved peak frequencies $f_{1,2}$ are clearly visible at around 10 and $70 \mathrm{GHz}$, respectively. The time-domain data were fitted with a sum of two decaying sinusoidal functions $y_{i}(i=1,2)$ :

$$
y_{i}=A_{i} \exp \left(-t / \tau_{i}\right) \sin \left(2 \pi t f_{i}\right),
$$


where $A_{i}, \tau_{i}$, and $f_{i}$ are fitting parameters. The result of the fit is shown by the solid line in Fig. 1(a) and yields $f_{1}=10.6 \pm 0.1$ and $f_{2}=70.2 \pm 0.6 \mathrm{GHz}$, respectively, which are in good agreement with the frequencies extracted from the FFT.

The effect of an external magnetic field on both oscillation modes was examined in the region of the sample with $79.5 \% \mathrm{Co}$, with the external magnetic field applied at an angle $\approx 55^{\circ}$ from the sample normal. The peak frequencies as a function of the external magnetic field are plotted in Fig. 1(b). $f_{1}$ increases linearly with the applied field, whereas $f_{2}$ does not depend on magnetic field. Such observations are in accordance with the behavior expected for ferromagnetic and exchange modes, respectively, [see Eqs. (4) and (6)]. The dependences of both frequencies on Co concentration are plotted in Figs. 1(c) and 1(d). The solid lines are guides to the eye. At a certain Co concentration $\left(81.5 \%\right.$ Co) $f_{1}$ reaches a maximum whereas $f_{2}$ is at its minimum value. Again, this is in good qualitative agreement with what is theoretically expected at AMC for ferromagnetic and exchange modes, respectively, according to Eqs. (4) to (6). Therefore, $f_{1}$ and $f_{2}$ are identified as the frequencies of ferromagnetic and exchange resonance modes, respectively. Accordingly, $81.5 \%$ Co is considered as corresponding to the composition at which the angular momentum of the system vanishes, i.e., the AMC composition at room temperature. The ways the modes oscillate are shown by cartoonlike schematics in Figs. 1(e) and 1(f). The FMR mode corresponds to a precession of the Co and Gd moments around the net effective field $\mathbf{H}_{\text {eff }}$, while staying rigidly antiferromagnetically coupled to each other [Fig. 1(e)]. In the case of the exchange mode, unequal action of the laser pulse light [20] on $\mathrm{Gd}$ and $\mathrm{Co}$ and two different precession rates $\left(\gamma_{\mathrm{Co}} \neq \gamma_{\mathrm{Gd}}\right)[24,25]$ induce a canting angle between the sublattices [Fig. 1(f)]. Consequently, the magnetizations precess around the exchange fields $\mathbf{H}_{\text {exch }}^{\mathrm{Co}, \mathrm{Gd}}$, respectively, which give rise to the exchange mode.

We have also performed similar measurements at room temperature, at a fixed composition of $78 \% \mathrm{Co}$, as a function of pump-laser fluence. The spin oscillations, the corresponding frequencies and resonance amplitudes are shown in the main panel and the inset of Fig. 2(a), and in Fig. 2(b), respectively. Excitation of the ferromagnetic and exchange modes is shown to be strongly fluence dependent and three different regimes of excitation can be distinguished. When the pump-laser fluence is low [regime (I)] only the FMR mode is excited and the amplitude of the spin oscillations increases with fluence [Fig. 2(b)]. When the fluence reaches $2.5 \mathrm{~mJ} / \mathrm{cm}^{2}$ the laser light starts to excite the exchange mode as well, while the amplitude of the FMR mode experiences a significant drop [regime (II)]. In this regime the exchange and the FMR modes are excited with nearly equal amplitudes. For the pump fluence at $3.6 \mathrm{~mJ} / \mathrm{cm}^{2}$ the frequency of the FMR mode is
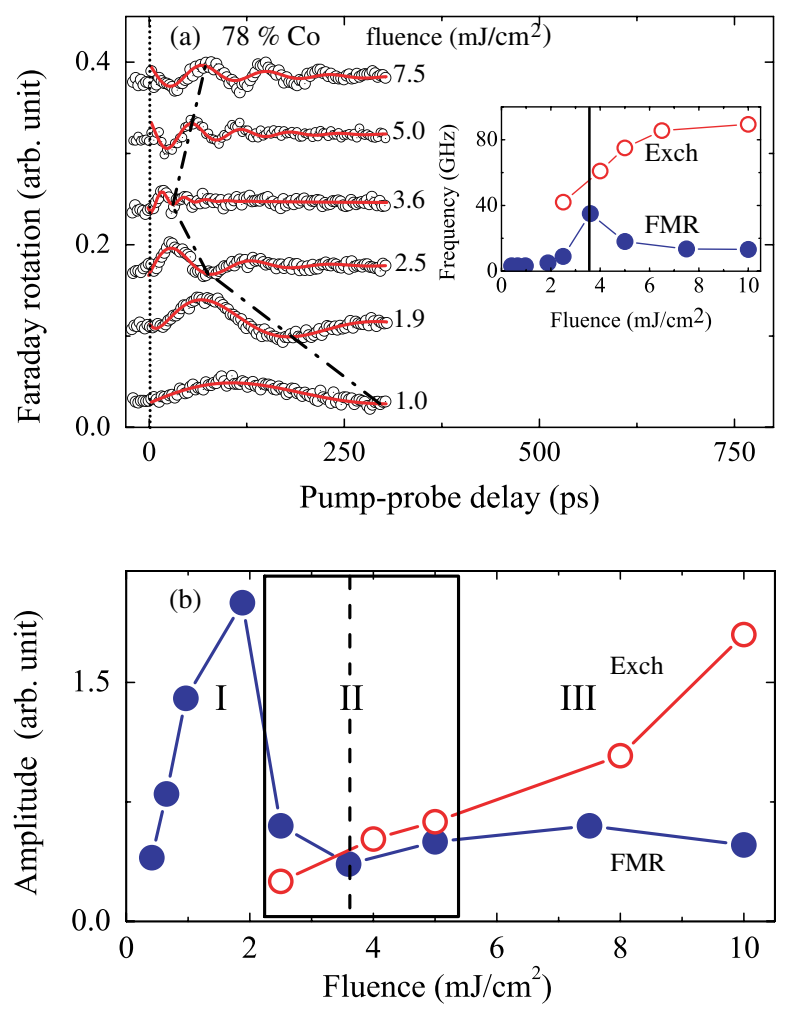

FIG. 2 (color online). (a) Magnetic oscillations corresponding to the FMR mode for $78 \%$ Co as a function of pump-laser fluence. The solid curves are fits using function $y_{1}$ [Eq. (7)], and the dash-dotted line is a guide to the eye that shows the evolution of the FMR mode's period. Frequencies of the FMR and exchange modes as a function of pump-laser fluence are shown as inset. (b) Resonance amplitudes of the two modes as a function of pump-laser fluence.

maximum [inset of Fig. 2(a)] while the amplitude has a minimum [Fig. 2(b)]. For a higher fluence the phase of the oscillation corresponding to the FMR mode is reversed [main panel of Fig. 2(a)]. These findings indicate that a laser excitation with $3.6 \mathrm{~mJ} / \mathrm{cm}^{2}$ brings the system to the AMC point: for fluences beyond this, the laser excitation reverses the sublattices' magnetizations, in the presence of an external field, by increasing the spin temperature beyond the compensation temperature. Finally, in the highfluence regime (III), the amplitude of the exchange mode increases with laser fluence, while the amplitude of the FMR mode hardly changes [Fig. 2(b)].

These observations allow us to draw important conclusions about the roles of the FMR and exchange modes in the optical control of magnetism. If the sample is below its AMC temperature and the pump fluence is low enough, the pump pulse brings the medium into a state with a higher temperature but still below the compensation point. As it has been demonstrated previously [4,26-28], such an ultrafast heating favors the excitation of the FMR mode [regime (I)]. Our experiments also clearly show that oscillations at the exchange frequency cannot be triggered in 
this regime. It appears that crossing the compensation point is crucial for the excitation of the exchange mode. In a quasistatic situation, heating GdCo over the compensation point in an external magnetic field should result in the reversal of the sublattices magnetizations. However, it has been recently discovered that the rare-earth and transition metal sublattices reverse their magnetizations at substantially different time scales [10]. Consequently, despite the strong antiferromagnetic coupling between the sublattices, the reversal occurs via a transient ferromagneticlike state, where the net magnetizations of the sublattices are temporarily aligned parallel to each other. The strong laser-pulse perturbation of the antiparallel alignment of the magnetizations can cause the creation of a larger angle between the exchange fields and the magnetizations [Fig. 1(f)]. Such condition makes the exchange mode resonate with larger amplitude. Therefore, the formation of a transient ferromagneticlike state in the time window where spin dynamics is initiated obviously favors the excitation of the exchange mode, while the excitation of the FMR mode becomes less effective.

In conclusion, using optical pulses, the high-frequency exchange and low-frequency ferromagnetic spin resonance modes have been excited and detected in a $\mathrm{Gd}_{1-x} \mathrm{Co}_{x}$ ferrimagnetic thin film on both sides of its angular momentum compensation composition. We have revealed ways to excite selectively, efficiently, and controllably either one or both of these two coexisting magnetic modes by adjusting the pump-laser fluence, and thus the temperature of the spin reservoir in the pumped area of the sample as compared to its angular momentum compensation temperature. Additionally, laser-heating-induced switching of the sublattices' magnetizations by increasing the spin temperature beyond the compensation temperature has been demonstrated. Such easy all-optical manipulation of the exchange mode in ferrimagnetic GdCo may give great opportunities for the application of this system in the field of ultrafast, laser-controlled addressing of magnetic information.

We thank A. J. Toonen and A. F. van Etteger for technical support. We acknowledge financial support from the Netherlands Organization for Scientific Research (NWO), de Stichting voor Fundamenteel Onderzoek der Materie (FOM), the European Community's Seventh Framework Program (FP7/2007-2013), under Grants NMP3-SL-2008214469 (UltraMagnetron) and N 214810 (FANTOMAS), and the French Fondation Nanoscience and ANR-07NANO-034 Dynawall project.

[1] E. Beaurepaire, J.-C. Merle, A. Daunois, and J.-Y. Bigot, Phys. Rev. Lett. 76, 4250 (1996).
[2] C. H. Back, D. Weller, J. Heidmann, D. Mauri, D. Guarisco, E. L. Garwin, and H. C. Siegmann, Phys. Rev. Lett. 81, 3251 (1998).

[3] C. H. Back, R. Allenspach, W. Weber, S. S. P. Parkin, D. Weller, E. L. Garwin, and H. C. Siegmann, Science 285, 864 (1999).

[4] M. van Kampen, C. Jozsa, J. T. Kohlhepp, P. LeClair, L. Lagae, W. J. M. de Jonge, and B. Koopmans, Phys. Rev. Lett. 88, 227201 (2002).

[5] T. Gerrits, H. A. M. van den Berg, J. Hohlfeld, L. Bar, and T. Rasing, Nature (London) 418, 509 (2002).

[6] A. V. Kimel, A. Kirilyuk, A. Tsvetkov, R. V. Pisarev, and T. Rasing, Nature (London) 429, 850 (2004).

[7] A. V. Kimel, A. Kirilyuk, P. A. Usachev, R. V. Pisarev, A. M. Balbashov, and T. Rasing, Nature (London) 435, 655 (2005).

[8] C.D. Stanciu, F. Hansteen, A. V. Kimel, A. Kirilyuk, A. Tsukamoto, A. Itoh, and T. Rasing, Phys. Rev. Lett. 99, 047601 (2007).

[9] K. Vahaplar, A. M. Kalashnikova, A. V. Kimel, D. Hinzke, U. Nowak, R. Chantrell, A. Tsukamoto, A. Itoh, A. Kirilyuk, and T. Rasing, Phys. Rev. Lett. 103, 117201 (2009).

[10] I. Radu et al., Nature (London) 472, 205 (2011).

[11] A. Hrabec, N. T. Nam, S. Pizzini, and L. Ranno Appl. Phys. Lett. 99, 052507 (2011).

[12] P. Chaudhari, J. J. Cuomo, and R. J. Gambino, Appl. Phys. Lett. 22, 337 (1973).

[13] R. C. Taylor and A. Gangulee, J. Appl. Phys. 47, 4666 (1976).

[14] M. Mansuripur, The Physical Principles of MagnetoOptical Recording (Cambridge University Press, Cambridge, England, 1995).

[15] L. Landau and E. Lifshitz, Phys. Z. Sowjetunion 8, 153 (1935), http://ujp.bitp.kiev.ua/files/file/papers/53/special_ issue/53SI06p.pdf.

[16] T. L. Gilbert, IEEE Trans. Magn. 40, 3443 (2004).

[17] R. K. Wangsness, Phys. Rev. 91, 1085 (1953).

[18] J. Kaplan and C. Kittel, J. Chem. Phys. 21, 760 (1953).

[19] R. K. Wangsness, Phys. Rev. 97, 831 (1955).

[20] S. S. Jaswal, D. J. Sellmyer, M. Engelhardt, Z. Zhao, A. J. Arko, and K. Xie, Phys. Rev. B 35, 996 (1987).

[21] P. Hansen, C. Clausen, G. Much, M. Rosenkranz, and K. Witter, J. Appl. Phys. 66, 756 (1989).

[22] N. H. Duc and D. Givord, J. Magn. Magn. Mater. 157-158, 169 (1996).

[23] T. A. Ostler et al., Phys. Rev. B 84, 024407 (2011).

[24] E. P. Wohlfahrt, Ferromagnetic Materials (North Holland, Amsterdam, 1980), Vol. 1.

[25] J. Pelzl, R. Meckenstock, D. Spoddig, F. Schreiber, J. Pflaum, and Z. Frait, J. Phys. Condens. Matter 15, S451 (2003).

[26] C. D. Stanciu, A. V. Kimel, F. Hansteen, A. Tsukamoto, A. Itoh, A. Kirilyuk, and T. Rasing, Phys. Rev. B 73, 220402 (2006).

[27] M. Binder et al., Phys. Rev. B 74, 134404 (2006).

[28] A. Tsukamoto, T. Sato, S. Toriumi, and A. Itoh, J. Appl. Phys. 109, 07D302 (2011). 